\title{
Migraine and Genetic Polymorphisms: An Overview
}

\author{
Vincenzo Pizza ${ }^{1}$, Anella Agresta ${ }^{1}$, Antonio Agresta ${ }^{1}$ Eros Lamaida ${ }^{1}$, Norman Lamaida ${ }^{1}$, Francesco \\ Infante $^{2}$ and Anna Capasso $3, *$
}

\author{
${ }^{1}$ Neurophysiopathology Service, S. Luca Hospital, Vallo della Lucania (SA), Italy \\ ${ }^{2}$ Biochemical Laboratory, S. Luca Hospital, Vallo della Lucania (SA), Italy \\ ${ }^{3}$ Department of Pharmaceutical and Biomedical Sciences, University of Salerno, Italy
}

\begin{abstract}
The relationship between genetic polymorphisms and migraine as a cause of an increased risk of thrombotic disorders development is still debated

In this respect, factor V Leiden, factor V (H1299R), prothrombin G20210A, factor XIII (V34L), $\beta$-fibrinogen, MTHFR (C677T), MTHFR (A1298C), APO E, PAI-1, HPA-1 and ACE I/D seem to play a determinant role in vascular diseases related to migraine.
\end{abstract}

The present review analyzes both the incidence of the above genetic vascular mutations in migraineurs and the most recent developments related to genetic polymorphisms and migraine.

Keywords: Genetic Polymorphisms, Migraine, Thrombosis, Vascular Disease.

\section{INTRODUCTION}

Migraine is a common neurovascular disorder. During the last decade, many clinical studies have given evidence of an association between migraine, particularly migraine with aura, and ischaemic stroke. There are several pathophysiological mechanisms implicated in the genesis of ischaemic events in migrainous patients [1].

Recent studies assess the role of migraine as a risk factor for endothelial dysfunction, responsible not only for a reduced availability of vasodilatators and for an increase of vasoconstrictor agents, but also for a release of procoagulant, proinflammatory and proliferative factors, predisposing migraineurs to atherogenesis [2].

Endothelial dysfunction is due to an increased oxidative stress, promotor of inflammatory processes, proposed as implicated in the pathogenesis of migraine [3].

Migraine is related to an intracranial vasospasm followed by the maximum dilatation of extra and intra-cranial arteries responsible for pain [2-4]. Considering that platelet activation and plasma coagulability are increased during migraine attacks [5], it is strongly supported that genes affecting vascular endothelial function could play a significant role in cerebral blood flow changes occurring in migraineurs, so, contributing to the aetiology of the migraine [5,6].

In this respect, factor $\mathrm{V}$ Leiden, factor $\mathrm{V}$ (H1299R), prothrombin G20210A, factor XIII (V34L), $\beta$-fibrinogen, MTHFR (C677T), MTHFR (A1298C), APO E, PAI-1, HPA1 and ACE I/D genetic polymorphisms seem to play a determinant role in vascular diseases in migraine [2-6].

*Address correspondence to this author at the Department of Pharmaceutical and Biomedical Sciences, University of Salerno, Italy;

Tel: +39 089 969744; Fax: +39 089 966262; E-mail: annacap@unisa.it
Therefore, the association between genetic polymorphisms and migraine may induce an increased risk of thrombotic disorders development [2-6].

The present review analyzes both the incidence of the above genetic vascular mutations in migraineurs and the most recent developments related to genetic polymorphisms and migraine.

\section{MIGRAINE AND GENETIC POLYMORPHISMS}

Recently, several angiotensin I-converting enzyme (ACE) inhibitors and an angiotensin II receptor blocker were demonstrated to have a clinically important prophylactic effect in migraine. ACE is one of the key enzymes in the reninangiotensin-aldosterone system, which modulates vascular tension and blood pressure [7]. In humans, serum ACE levels are strongly genetically determined. Individuals who were homozygous for the deletion (D) allele showed increased ACE activity levels. To investigate the role of ACE polymorphism in headache, the ACE insertion (I)/deletion (D) genotypes of 54 patients suffering from migraine with aura (MwA), 122 from migraine without aura, 78 from tensiontype headache (TH), and 248 non-headache healthy controls was studied by Kowa et al. [7]. The ACE D allele were significantly more frequent in the MwA than controls. The incidence of the D/D genotype in MwA (25.9\%) was significantly higher than that in controls $(12.5 \%)$. No differences in the remaining groups were found. These findings indicate that the $\mathrm{D}$ allele and the $\mathrm{D} / \mathrm{D}$ genotype in the ACE gene is a genetic risk factor for MwA thus suggesting a possible relationship between ACE activity and the pathogenesis of migraine [7].

Paterna et al. [8] evaluate if the DD genotype could also be associated with the frequency and duration of migraine 
without aura. In 302 patients suffering from migraine without aura, the genotypes of the ACE gene, plasma ACE activity, and the frequency (weekly) and duration of migraine attacks were evaluated. No drugs were given before (4 weeks) and during the study. The same evaluations were performed in 201 subjects without migraine. Patients with migraine without aura showed higher incidence of the ACE-DD gene (48.34\%) than control subjects $(37.32 \%)$. The frequency of migraine (average attacks per week) was higher in patients with DD $(2.11+/-1.9)$ than in patients with ID $(1.54+/-1$. 44). No difference in duration of migraine attacks (hours per week) was observed. Plasma ACE activity was increased in patients with the ACE-DD gene. These data suggest that ACE-DD gene polymorphism could have an important role in determining migraine attacks and the frequency of these attacks [8].

Also, angiotensin converting enzyme (ACE) gene has been implicated as a genetic factor associated with migraine [9]. A case-control study was performed to investigate the association between ACE and migraine in 240 migraine patients and 200 healthy controls [9]. There was no significant difference in allelic frequency (I and D) and genotype polymorphism (DD, DI and II) of the ACE gene in migraine patients and controls. Analysis of the difference in ACE polymorphism stratified by gender revealed that male migraine patients with the homozygote DD genotype (ACE-DD) were significantly fewer than that of male controls. There was no existence of a difference among the frequency and duration of headache in each subgroup of migraine patients stratified by ACE genotype. These findings indicate that ACE-DD may have a slight protective effect against migraine in male patients [9].

Another study was conducted to determine whether the ACE I/D gene variant is involved in migraine risk and whether this variant might act in combination with the previously implicated MTHFR C677T genetic variant in $270 \mathrm{mi}-$ graine cases and 270 matched controls [10]. Statistical analysis of the ACE I/D variant indicated no significant difference in allele or genotype frequencies. However, grouping of genotypes showed a modest, yet significant, over-representation of the DD/ID genotype in the migraine group (88\%) compared to controls (81\%). Multivariate analysis, including genotype data for the MTHFR C677T, provided evidence that the MTHFR (TT) and ACE (ID/DD) genotypes act in combination to increase migraine susceptibility [10]. This effect was greatest for the MA subtype where the genotype combination corresponded to an OR of 2.89 (95\% CI:1.47$5.72, \mathrm{P}=0.002$ ). In Caucasians, the ACE D allele confers a weak independent risk to migraine susceptibility and also appears to act in combination with the C677T variant in the MTHFR gene to confer a stronger influence on the disease [10].

Even the role of factor XIII (V34L), APO E gene, MTHFR C677T and A1298C polymorphisms in migrainous patients with cerebrovascular risk was considered [11-16] for migraine's vulnerability.

In fact, the common Val34Leu polymorphism of the Achain factor XIII gene, associated with variations in factor XIII activity, has been suggested to play a significant role in the development of arterial and venous thrombotic disorders
[11]. It was analysed the prevalence of this polymorphism in 17 patients with coexisting ischaemic cerebrovascular disease and migraine (5 with aura, and 12 without aura), 89 patients with migraine (43 with aura, and 46 without aura), 116 patients with ischaemic cerebrovascular disease, and 467 healthy Caucasian controls [11]. The factor XIII Leu 34 variant was present in $47.1 ; 40.5 ; 34.9$; and $35.1 \%$ of patients with coexisting ischaemic cerebrovascular disease and migraine, ischaemic cerebrovascular disease, migraine, and control subjects, respectively. These data suggest that the factor XIII Leu 34 allele does not play a protective role against these disorders in our population [11].

Rainero et al. investigated the role of apolipoprotein $\mathrm{E}$ (APOE) polymorphisms in migraine by analyzing the APOE genotypes of 241 migraine patients and 587 controls [12]. The results indicated that APOE alleles were similarly distributed between cases and controls. However, a significant increase of the varepsilon2-varepsilon4 genotype in the group of migraine patients was also observed. Patients were divided into three subgroups: migraine with aura; migraine without aura; and mixed headaches (migraine associated with tension-type headache). Subgroup analysis showed that the varepsilon2-varepsilon4 genotype was significantly increased only in patients with mixed headaches. The stratification of patients by APOE status did not reveal significant associations with the clinical features of the disease. In conclusion, this study showed no significant association between APOE polymorphisms and migraine [12].

Another study [13] was performed to determine the prevalence of the MTHFR mutation in patients with migraine and tension-type headache (TH). Seventy-four patients with migraine headaches ( 22 with aura and 52 without aura), 47 with THs, and 261 normal controls were recruited. This study showed that the incidence of the homozygous transition $(\mathrm{T} / \mathrm{T})$ in migraine sufferers $(20.3 \%)$ was significantly higher than that in controls $(9.6 \%)$. Moreover, the frequency of the $\mathrm{T} / \mathrm{T}$ genotype in individuals with migraine headaches with aura was remarkably high $(40.9 \%)$. The MTHFR $T$ allele was more frequent in the migraine group than in the control group. Therefore, these results support the conclusion that the MTHFR gene, causing mild hyperhomocysteinemia, may be a genetic risk factor for migraine [13].

Finally, in a case-control study, was studied the prevalence of two common MTHFR polymorphisms, C677T and A1298C, in 102 migraine patients (23 migraine with aura, 70 migraine without aura and nine with tension-type headache) and compared it to that of 136 healthy controls [14]. The frequencies of the T allele of MTHFR677 and the C allele of MTHFR1298 were significantly higher in the total migraine population $(33.82 \%, 33.82 \%)$ than in controls $(25.38 \%$ and $24.26 \%$ ), respectively [14]. The genotypes T677T and C1298C were the only genotypes significantly associated with migraine. Patients with migraine with aura with C1298C and C677C/C1298C genotypes were even more profoundly associated with migraine risk than others [14]. However patients with migraine without aura with T677T and C1298C genotypes showed the same susceptibility. Patients with $\mathrm{C} 1298 \mathrm{C}$ and $\mathrm{C} 677 \mathrm{C} / \mathrm{C} 1298 \mathrm{C}$ genotypes may also predispose to tension-type headache. 
Although the above studies were conflicting, they suggested a higher frequency of some prothrombotic genetic abnormalities in migrainous patients [11-14].

While the exact etiology of migraine headaches is unknown, several theories have been proposed. The vascular theory, as proposed in 1938 by Graham and Wolff [15], attributes migraines to an initial intra-cranial arterial vasoconstriction, resulting in reduced blood flow to the visual cortex, followed by a period of extra-cranial vasodilation [16]. Modern imaging techniques have shown that during a common migraine attack there are in fact only minor changes in cerebral blood flow, and the proposed initial vasoconstrictive phase may actually last much longer than the aura [17]. It has also been hypothesised that migraine sufferers have an inherent vasomotor instability and are more susceptible to the vasodilatory effects of certain physical and chemical agents. This point of view has been reinforced by the observation that organic nitrates, which are capable of delivering nitric oxide, trigger migraine attacks in migraineurs, at low doses, ineffective in normal subjects [18].

Moskowitz's theory involves the trigeminovascular complex, which links the aura and the headache of migraine [1920]. In this theory the trigeminovascular neurons release substance $\mathrm{P}$ and other neuro-transmitters in response to various triggers (Fig. 1).

Taken together, these events configure the so-called phenomenon of "neurogenic inflammation" considering that both excessive trigeminal discharge and neurovascular in- flammation of meninges occur in migraine headache.

The relationship between hypercoagulability state and migraine is another interesting pathogenetic aspect to be considered. In this regard homocysteinemia is believed to be a clear risk factor for thrombosis and it can be associated with an increased stroke and cardiovascular risk [16-20].

Vascular disease seems to be mediated by a growth of oxidative stress that, in its turn, could show the way to vascular disease by direct and indirect mechanisms [21].

A direct association has been shown between oxidative plasmatic capacity and homocysteine plasmatic levels. Among these being the production of endothelial nitric oxide, one of the most important risk factors for vascular disease and, at the same time, a mediator involved in migraine genesis [22].

Hyperhomocysteinemia is an indipendent risk factor for coronary and artery disease $[23,24]$ and is also associated with cerebrovascular disease $[25,26]$. Multiple genetic and environmental factors can lead to hyperhomocysteinemia. In particular a polymorphism in the 5,10-methylenetetrahydrofolate reductase (MTHFR) gene has been described and individuals homozygous for the mutation C677T, who exhibit reduced enzymatic activity and elevated homocysteine concentration [27]. The decreased activity of MTHFR, in case of mutated enzyme, determines a reduced cellular availability of methyl THF, the substrate for methionine synthase. This enzyme catalyses the transfer of a methyl group from methylenetetrahydrofolate to homocysteine, producing THF and

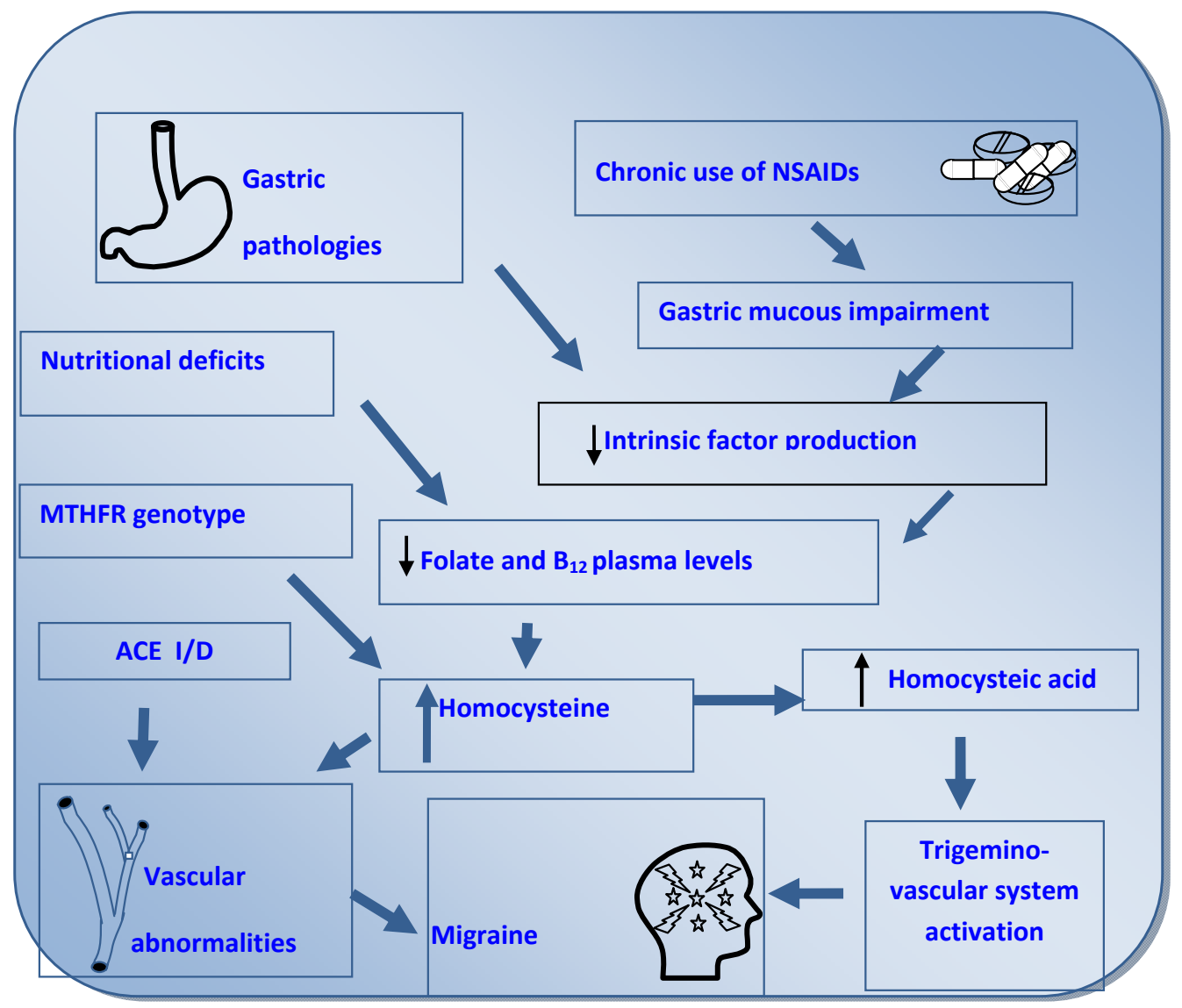

Fig. (1). Integrated pathogenetic model of migraine. 
methionine. By this way C677T mutation in MTHFR gene can lead to elevated homocysteine concentration. Two different studies have investigated the prevalence of this mutation in migraineurs patients [14,28]. The MTHFR $T$ allele seems to be more frequent in the migraine group than in the control group in both studies. Furthermore Kara et al. [14] determined the prevalence of another MTHFR polymorphism (A1298C) in migraine patients. The C allele of MTHFR1298 was significantly higher in migraine and tension-type headache patients. Interestingly migraine with aura patients with $\mathrm{C} 1298 \mathrm{C}$ and $\mathrm{C} 677 \mathrm{C} / \mathrm{C} 1298 \mathrm{C}$ genotypes were even more profoundly associated with migraine risk than others. The authors have not analysed blood homocysteine levels, but elevated serum levels of homocysteine have been reported in patients suffering of migraine with aura not in tension-type headache patients. Hering-Hanit et al [29] examined blood homocysteine in a significant number of patients with migraine but they did not find its levels augmented. To the best of our knowledge no study has been conducted on the possible correlation between blood homocysteine levels and migraine severity and on the possible role of hyperhomocysteinemia as a causative factor in the predisposition to migraine. In fact it is well known that hyperhomocysteinemia may lead to an excessive production of homocysteic acid [30]. In this view elevated homocysteic acid levels, secondary to hyperhomocysteinemia, could sensitize dura mater and cerebral arteries and/or favour the activation of trigeminovascular system, thus predisposing a patient to migraine attacks or increasing disease's severity [30].

However, an impairment of mitochondrial oxidative metabolism might also play a role in the pathophysiology of migraine, by influencing neuronal information processing. Biochemical assays of platelets and muscle biopsies performed in migraine sufferers have shown a decreased activity of the respiratory chain enzymes [31]. Studies with phosphorus magnetic resonance spectroscopy ((31)P-MRS) have demonstrated an impairment of the brain oxidative energy metabolism both during and between migraine attacks [31]. However, molecular genetic studies have not detected specific mitochondrial DNA (mtDNA) mutations in patients with migraine, although other studies suggest that particular genetic markers (i.e. neutral polymorphisms or secondary mtDNA mutations) might be present in some migraine sufferers [31].

Also, mitochondrial DNA (mtDNA) haplogroup U, defined by the polymorphism $12308 \mathrm{~A}>\mathrm{G}$, may constitute a risk factor for a stroke in migraine [31]. Finnila et al. [32] identified 14 patients with an occipital stroke and with $12308 \mathrm{~A}>\mathrm{G}$ by determining complete mtDNA coding region sequence for the patients and for population controls by conformation sensitive gel electrophoresis (CSGE) and direct sequencing [32]. Sequence information was used to construct a phylogenetic network of mtDNA haplogroups $\mathrm{U}$ and $\mathrm{K}$, which was found to be composed of subclusters U2, U4, U5 and a new subcluster U7, as well as cluster K. Five patients with a migrainous stroke belonged to subcluster U5 ( $\mathrm{P}=0.006$; Fisher's exact test). Many unique mutations were found among the patients with an occipital stroke including two tRNA mutations that have previously been suggested to be pathogenic. Analysis of mtDNA sequences by CSGE and comparison of the sequences through phylogenetic analysis greatly en- hances the identification of mtDNA clusters in population and detection of mtDNA mutations in patients [32].

In this respect, it is very interesting the link between migraine, mitochondrial pathology and drugs used in relation to particular genetic mutations. The association between migraine and abnormal mitochondrial function has been suggested by clinical similarities between this form of headache and some mitochondrial diseases such as MELAS [33] in which 10 different mutations have been identified, but $80 \%$ of cases the mutation is $3243 \mathrm{~A}>\mathrm{G}$ in the leucine tRNA gene (tRNA Leu) and it is typically associated with migraine attacks with aura, vomiting and acute migraine. The mutation is transmitted by maternal inheritance. Other examples are MERRF and NARP [34]. MERRF syndrome (myoclonic epilepsy with ragged red fibers) is a mitochondrial encephalomyopathy, characterized by myoclonic seizures in combination, sometimes, sensorineural deafness, optic atrophy, short stature, or peripheral neuropathy. In some cases were observed lipomatosis, cardiomyopathy, retinitis pigmentosus, ophthalmoparesis and / or pyramidal signs. The disease is progressive, with worsening of epilepsy and other symptoms, such as ataxia, deafness, muscle weakness and dementia. Over $80 \%$ of patients present with the mutation $8344 \mathrm{~A}>\mathrm{G}$ in the gene coding for the RNA transport of lysine (MTTK). Have also been identified other mutations in other genes coding for RNA transport and in the gene MTND5. These mutations can cause overlapping clinical syndrome (MERRF / MELAS) with infarct-like episodes.

Neuropathy, ataxia, and retinitis pigmentosus (NARP) is a condition that causes a variety of signs and symptoms affecting the nervous system. NARP, is a condition related to changes in mitochondrial DNA with a mutations in the MTATP6 gene.

Of more recent interest is the identification of haplogroups of the mitochondrial genome related to migraine. Zaki et al [35] studied the entire mitochondrial genome and sequenced in 20 haplogroup-H patients with migraine without aura (MoA), syndrome vomiting cicling (CVS) e controls. Polymorphisms of interest were tested in 10 additional CVS subjects and in 112 haplogroup-H adults with MoA. The $16519 \mathrm{C}-->\mathrm{T}$ polymorphism was found to be highly disease associated: $21 / 30$ CVS subjects [70\%, odds ratio (OR) $6.2]$ and $58 / 112$ migraineurs (52\%, OR 3.6$)$ vs. $63 / 231$ controls (27\%). A second polymorphism, 3010G-->A, was found to be highly disease associated in those subjects with 16519T: 6/21 CVS subjects (29\%, OR 17) and 15/58 migraineurs $(26 \%$, OR 15$)$ vs. $1 / 63$ controls $(1.6 \%)$. The authors concluded that these polymorphisms constitute a substantial proportion of the genetic factor in migraine pathogenesis, and strengthen the hypothesis that there is a component of mitochondrial dysfunction in migraine.

Also, it was attempted to correlate a particular haplogroup to the therapeutic response, Di Lorenzo et al [36] tested in sixty-four migraineurs if the therapeutic response to riboflavin is associated with specific mitochondrial DNA (mtDNA) haplogroups, particurarly, they focused their attention on haplogroup $\mathrm{H}$, which is known to differ from others in terms of energy metabolism. Forty patients responded to riboflavin treatment and 24 were nonresponders. The mtDNA haplogroup $\mathrm{H}$ was found in 29 subjects (20 migraine 
without aura, 9 migraine with aura). Riboflavin responders were more numerous in the non-H group $(67.5 \%)$. Conversely, nonresponders were mostly $\mathrm{H}(66.7 \%)$. The difference between the two groups was significant $\left(\chi^{2}=7.07 ; p=\right.$ 0.01 ). The presence of aura had no influence on riboflavin's effectiveness $\left(\chi^{2}=0.113 ; p=0.74\right)$ and was not associated with a particular haplogroup $\left(\chi^{2}=0.55 ; p=0.46\right)$. In this pharmacogenetic study, riboflavin appears to be more effective in patients with migraine with non- $\mathrm{H}$ mitochondrial DNA haplotypes. The underlying mechanisms are unknown, but could be related to the association of haplogroup $\mathrm{H}$ with increased activity in complex I, which is a major target for riboflavin. These results may have ethnic implications, since haplogroup $\mathrm{H}$ is chiefly found in the European population.

\section{CONCLUSIONS}

The present review suggest that genetic polymorphism could have an important role in determining migraine attacks, and could also affect the frequency of these attacks. Considering the studies presented, it is possible to supplement the pathogenetical pattern of migraine with genetic polymorphism resulting also in an alteration of endothelial regulation tone as well as mitochondrial function. Therefore, we may conclude that genetic mutations play a crucial role in the development of migraine and in the increased risk to develop an ischaemic disease in migraineurs, but further studies are necessary. Certainly further data is needed, through further studies, especially on the biomolecular level and on the quantification of the relative risk for each polymorphism and for their combination. In the future, the genotype therapy could be considered as a diagnostic marker for therapy through the use of anti-migraine drugs. Thus, it could be possible to perform a primary prevention of this not very well known illness.

\section{CONFLICT OF INTEREST}

The authors confirm that this article content has no conflicts of interest.

\section{ACKNOWLEDGEMENTS}

None declared.

\section{REFERENCES}

[1] Lea RA, Ovcaric M, Sundholm J, MacMillan J, Griffiths LR. The methylenetetrahydrofolate reductase gene C677T influences susceptibility to migraine with aura. BMC Med 2004; 2: 3-7.

[2] Chiodini BD, Barlera S, Franzosi MG, Rognoni G. I geni di suscettibilità all'infarto: una revisione della letteratura. Ital Heart $\mathrm{J}$ 2001; 2: 935-944.

[3] C Colucci D'Amato V, Alfano E, Giordano T, Marmolo V. Pizza: Le Cefalee, Napoli: Idelson 1997, p.129.

[4] Bianchi A, Pitari GM, Amenta V, et al. Endothelial, Haemostatic and haemorheological modifications in migraineurs; Artery 1996; 22: 93-100.

[5] Corral J, Iniesta JA, Gonzà-Conejero R, Lozano ML, Rivera J, Vicente V. Migraine and prothrombotic genetic risk factors. Cephalalgia 1998; 18: 257

[6] Colson NJ, Fernandez F, Griffiths LR. Migraine genetics and prospects for pharmacotherapy. Drug Dev Res 2007; 68: 282-93.

[7] Kowa H, Fusayasu E, Ijiri I, et al. Association of the Insertion/Deletion polymorphism of the angiotensin I-converting enzyme gene in patients of migraine with aura. Neurosci Lett 2005; 374: $129-31$
[8] Paterna S, Di Pasquale P, D’Angelo A, et al. Angiotensin converting enzyme gene deletion polymorphism determines an increase in frequency of migraine attacks in patients suffering from migraine without aura. Eur Neurol 2000; 43: 133-43

[9] Lin JJ, Wang PJ, Chen $\mathrm{CH}$, et al. Homozygous deletion genotype of angiotensin converting enzyme confers protection against migraine in man. Acta Neurol Taiwan 2005; 14: 120-5.

[10] Lea RA, Ovcaric M, Sundholm J, Solyom L, MacMillan J, Griffiths LR. Genetic variants of angiotensin converting enzyme and methylenetetrahydrofolate reductase may act in combination to increase migraine susceptibility. Brain Res Mol Brain Res 2005; 136: 112-7.

[11] Iniesta JA, Corral J, Gonzà-Conejero R, et al. Role of factor XIII (Val $34 \mathrm{Leu}$ ) polymorphism in patients with migraine. Cephalalgia 2001; 8: 837-41.

[12] Rainero I, Grimaldi LM, Salani G, et al. Apolipoprotein E gene polymorphism in patients with migraine; Neurosci Lett 2002; 317: 111: 3 .

[13] Kowa H, Yasui K, Takeshima T, Urakami K, Sakai F, Nakashima $\mathrm{K}$. The homozygous C677T mutation in the methylenetetrahydrofolate reductase gene is a genetic risk factor for migraine. Neuropsychiatric Genet; 2000; 96: 762-4.

[14] Kara I, Sazei A, Ergul E, Kaya G, Kilie G. Association of the C677T and A1298C polymorphisms in the 5,10 methylenetetrahydrofolate reductase gene in patients with migraine risk. Brain Res Mol Brain Res 2003; 111: 84-90.

[15] Graham JR, Wolff HG. Mechanism of migraine headache and action of ergotamine tartrate. Arch Neurol Psychiatry 1938; 39: 73763.

[16] Wolff HG. Headache and Other Head Pain. $2^{\text {nd }}$ ed. New York: Oxford University Press 1963.

[17] Campbell JK, Caselli RJ. Headache and other craniofacial pain. In: Bradley WG, Daroff RB, Fenichel GM, Marsden CD, Eds. Neurology Clinical Prac 1991; 2: 1525-6. Butterworth-Heinemann, Boston.

[18] Thomsen LL, Iversen HK, Brinck TA, Olesen J. Arterial supersensitivity to nitric oxide (nitroglycerin) in migraine sufferers. Cephalalgia 1993; 13: 395-399.

[19] Moskowitz MA. The neurobiology of vascular head pain. Ann Neurol 1984; 16: 157-68.

[20] Moskowitz MA, Macfarlane R. Neurovascular and molecular mechanisms in migraine headaches. Cerebrovasc Brain Metab Rev 1993; 5: 159-77.

[21] Frosst P, Blom HJ, Milos R, et al. A candidate genetic risk factor for vascular disease: a common mutation in methylentetrahydrofolate reductase. Nat Genet 1995; 10: 111-3.

[22] Gemmati D. Le nuove mutazioni: Haemostasis e Thrombosis. Biochim Clin 2002; 26 (3): 135

[23] Bots ML, Launer LJ, Lindemans J, Hofman A. Grobbee DE. Homocysteine, atherosclerosis and prevalent cardiovascular disease in the elderly: The Rotterdam Study. J Intern Med 1999; 242: 339-47.

[24] Bostom AG, Silbershatz H, Rosenberg IH. Nonfasting plasma total homocysteine levels and all-cause and cardiovascular disease mortality in elderly Framingham men and women. Arch Intern Med 1999; 24: 1077-80.

[25] Selhub J, Jacques PF, Bostom AG, et al. Association between plasma homocysteine concentrations and extracranial carotid-artery stenosis. New Engl J Med 1995; 332: 286-91.

[26] Perry IJ. Refsum H, Morris RW, Ebrahim SB, Ueland PM, Shaper AG. Prospective study of serum total homocysteine concentration and risk of stroke in middle-aged British men. Lancet 1995; 346: 1395-8.

[27] Frosst P, Blom HJ, Milos R, et al. A candidate genetic risk factor for vascular disease: a common mutation in methylenetetrahydrofolate reductase. Nat Genet $1995 ; 10: 111-3$.

[28] Kowa H, Yasui K, Takeshima T, Urakami K, Sakai F, Nakashima $\mathrm{K}$. The homozygous C677T mutation in the methylenetetrahydrofolate reductase gene is a genetic risk factor for migraine. Am J Med Genet 2000; 96: 762-4.

[29] Hering-Hanit R, Gadoth N, Yavetz A, Gavendo S, Sala, B. Is blood hmocysteine elevated in migraine? Headache 2001; 41: 779-81.

[30] Wolff PA, Schaefer EJ, Rosenberg IH. Association between plasma homocysteine concentrations and extracranial carotid-artery stenosis. New Engl J Med 1995; 332: 286-91. 
[31] Sparaco M, Feleppa M, Lipton RB, Rapoport AM, Bigal ME. Mitochondrial dysfunction and migraine: evidence and hypotheses. Cephalalgia 2006; 26: 361-72.

[32] Finnilä S, Hassinen IE, Majamaa K. Phylogenetic analysis of mitochondrial DNA in patients with an occipital stroke. Evaluation of mutations by using sequence data on the entire coding region. Mutat Res 2001; 458: 31-9.

[33] Hansrote S, Croul S, Selak M, Kalman B, Schwartzmann RJ. External ophtalmologia with severe progressive multiorgan involvement associated with the mtDNA A3243G mutation. J Neurol Sci 2002; 197: 63-7
[34] Pietrobon D. Calcium channel and channelopathies of the central nervous system. Mol Neurobiol 2002; 25: 31-50

[35] Zaki EA, Freilinger T, Klopstock T, et al. Two common mitochondrial DNA polymorphisms are highly associated with migraine headache and cyclic vomiting syndrome. Cephalalgia 2009; 29: 719-28.

[36] Di Lorenzo C, Pierelli F, Coppola G, et al. Mitochondrial DNA haplogroups influence the therapeutic response to riboflavin in migraineurs. Neurology 2009; 72: 1588-94.

(C) Finsterer and Stöllberger; Licensee Bentham Open.

This is an open access article licensed under the terms of the Creative Commons Attribution Non-Commercial License (http://creativecommons.org/licenses/by-nc/3.0/) which permits unrestricted, non-commercial use, distribution and reproduction in any medium, provided the work is properly cited. 